\title{
Copper Tolerance Mediated by FgAceA and FgCrpA in Fusarium graminearum
}

\author{
Xin Liu ${ }^{1,2,3+}$, Yichen Jiang ${ }^{1,4+}$, Dan $\mathrm{He}^{1}$, Xin Fang ${ }^{1,3}$, Jianhong $X \mathrm{u}^{1,3}$, Yin-Won Lee ${ }^{1,5}$, \\ Nancy P. Keller ${ }^{2,6 *}$ and Jianrong Shi, ${ }^{1,3 *}$
}

\begin{abstract}
1 Jiangsu Key Laboratory for Food Quality and Safety-State Key Laboratory Cultivation Base, Ministry of Science and Technology/Key Laboratory for Control Technology and Standard for Agro-product Safety and Quality, Ministry of Agriculture and Rural Affairs/Key Laboratory for Agro-product Safety Risk Evaluation (Naniing), Ministry of Agriculture and Rural Affairs/Collaborative Innovation Center for Modern Grain Circulation and Safety/Institute of Food Safety and Nutrition, Jiangsu Academy of Agricultural Sciences, Nanjing, China, ${ }^{2}$ Department of Medical Microbiology and Immunology, University of Wisconsin-Madison, Madison, WI, United States, ${ }^{3}$ School of Food and Biological Engineering, Jiangsu University, Zhenjiang, China, ${ }^{4}$ College of Food Science, Tibet Agriculture and Animal Husbandry University, Nyingchi, China, ${ }^{5}$ School of Agricultural Biotechnology, Seoul National University, Seoul, South Korea, ${ }^{6}$ Department of Bacteriology, University of Wisconsin-Madison, Madison, WI, United States
\end{abstract}

\section{OPEN ACCESS}

Edited by:

Daisuke Hagiwara,

University of Tsukuba, Japan

Reviewed by:

Jinxing Song,

Jiangsu Normal University, China

Yun Chen,

Zhejiang University, China

${ }^{*}$ Correspondence:

Jianrong Sh

shijijaas.ac.cn

Nancy P. Keller

npkeller@wisc.edu

${ }^{t}$ These authors have contributed equally to this work

Specialty section:

This article was submitted to Fungi and Their Interactions,

a section of the journal

Frontiers in Microbiology

Received: 03 April 2020

Accepted: 29 May 2020

Published: 26 June 2020

Citation:

Liu X, Jiang Y, He D, Fang $X, X u$ J,

Lee $Y$-W, Keller NP and Shi J (2020)

Copper Tolerance Mediated by

FgAceA and FgCrpA in Fusarium

graminearum

Front. Microbiol. 11:1392.

doi: 10.3389/fmicb.2020.01392
All organisms must secure essential trace elements (e.g., Cu) for survival and reproduction. However, excess trace element accumulation in cells is highly toxic. The maintenance of copper (Cu) homeostasis has been extensively studied in mammals, bacteria, and yeast but not in plant pathogens. In this study, we investigated the molecular mechanisms of copper tolerance in Fusarium graminearum, the important wheat head scab fungus. RNA-seq revealed induced expression of the P-type ATPase transporter FgCrpA and metallothionein (MT) FgCrdA after excess Cu treatment. Deletion of $F g C r p A$ but not $F g C r d A$ resulted in reduced tolerance to $\mathrm{Cu}$ toxicity. The "Cu fist" transcription factor FgAceA was involved in Cu detoxification through activation of FgCrpA. $\triangle \mathrm{FgAceA}$ was more sensitive to copper toxicity than $\triangle \mathrm{FgCrpA}$ and overexpression of $F g C r p A$ restored copper tolerance in $\triangle \mathrm{FgAceA}$. FgAceA negatively regulated aurofusarin production and its biosynthetic gene expression. $\triangle \mathrm{FgCrpA}$ and $\triangle$ FgAceA were reduced in virulence in flowering wheat heads and synthesized decreased amounts of the mycotoxin deoxynivalenol when challenged with excess $\mathrm{Cu}$. Taken together, these results suggest that mediation of $\mathrm{Cu}$ tolerance in $F$. graminearum mainly relies on the Cu efflux pump and that FgAceA governs Cu detoxification through activation of FgCrpA.

Keywords: Fusarium graminearum, copper tolerance, FgAceA, copper transporters, metallothionein, virulence

\section{INTRODUCTION}

Copper $(\mathrm{Cu})$ is an essential trace element that cycles between $\mathrm{Cu}^{1+}$ (reduced) and $\mathrm{Cu}^{2+}$ (oxidized) states in virtually all organisms (Dalecki et al., 2017; Gerwien et al., 2018). By coordinating with proteins and serving as a redox cofactor, $\mathrm{Cu}$ confers changes in protein structure, catalytic activity and protein-protein interaction, thus controlling a variety of cellular biochemical processes (Kim et al., 2008). However, these same properties can be highly detrimental to microorganisms when 
$\mathrm{Cu}$ is present in excess. Free $\mathrm{Cu}$ can lead to oxidative stress by reacting with reactive oxygen species (ROS) to generate hydroxyl radicals that damage many biomolecules, including DNA, membrane lipids and proteins (Kim et al., 2008). Furthermore, $\mathrm{Cu}$ can disrupt the active sites of metallothioneins (MTs), such as iron-sulfur enzymes, and displace other metals from their cognate enzymes, leading to their inactivation (Besold et al., 2016).

Given its toxic properties to microorganisms, $\mathrm{Cu}$-containing compounds have been historically used as antimicrobials both in healthcare and agriculture (Dollwet and Sorenson, 1985; Grass et al., 2011; Festa et al., 2014; Michels et al., 2015). There is an emerging concept in innate immunity that animal hosts intentionally exploit copper toxicity as a weapon to combat invading microbes. For example, macrophages can attack invading microbes with high $\mathrm{Cu}$ levels, and $\mathrm{Cu}$ is elevated at sites in the lung and serum during infection (Besold et al., 2016).

In the host-microbe battle, on the other hand, the microbial intruder is equipped with a battery of $\mathrm{Cu}$ detoxification defenses that promotes survival and colonization in the host, including $\mathrm{Cu}$ binding MTs and $\mathrm{Cu}$ exporting P-type ATPases to defend against toxic levels of $\mathrm{Cu}$. MTs are cysteine (Cys)-rich low molecular weight polypeptides found in organisms from prokaryotes to mammals (Ding et al., 2014). MTs are utilized in response to high $\mathrm{Cu}$ levels and detoxify $\mathrm{Cu}$ through binding the $\mathrm{Cys}$ of $\mathrm{Cu}$ via Cysthiolate bonds (Palacios et al., 2011; Babula et al., 2012). P-type ATPases are heavy metal translocators conserved in all biological kingdoms that regulate intercellular $\mathrm{Cu}$ levels (Odermatt et al., 1993; Solioz and Odermatt, 1995; Ladomersky and Petris, 2015; Antsotegi-Uskola et al., 2017; Wiemann et al., 2017).

In the model yeast Saccharomyces cerevisiae, the metalloregulation of the $\mathrm{Cu}$-binding transcription factor (TF) Ace1 functions in Cu-replete cells and regulates the expression of $\mathrm{Cu}$ detoxification-related genes, including those encoding MTs (Cup1 and Crs5) and $\mathrm{Cu} / \mathrm{Zn}$ super oxide dismutase, SOD (SOD1) under excess $\mathrm{Cu}$ conditions (Keller et al., 2005; Cyert and Philpott, 2013). In the human fungal pathogen Cryptococcus neoformans, CMT1 and CMT2 genes encode cysteine-rich Cu-binding MTs, whose expression levels are dramatically upregulated in response to elevated $\mathrm{Cu}$ levels, play essential roles in $\mathrm{Cu}$ detoxification (Ding et al., 2011) and are critical for fungal virulence (Ding et al., 2013). While MTs have been proven to be the major players in $\mathrm{Cu}$ detoxification in S. cerevisiae and C. neoformans, P-type ATPases that pump $\mathrm{Cu}$ extracellularly through the transmembrane channel are utilized for $\mathrm{Cu}$ export in Candida albicans and Aspergillus spp. In the pathogenic yeast $C$. albicans, CRP1, which encodes a plasma membrane-localized $\mathrm{Cu}$ exporter P-type ATPase (Crp1), is transcriptionally upregulated by high $\mathrm{Cu}$ conditions in an Ace1-dependent manner, and deletion of CRP1 resulted in a $\mathrm{Cu}$-sensitive growth phenotype as well as massive intracellular $\mathrm{Cu}$ accumulation (Weissman et al., 2000). Recent studies have shown that $A$. fumigatus, A. nidulans, and A. flavus share a similar $\mathrm{Cu}$ detoxification machinery; under excess $\mathrm{Cu}$ conditions, the Cu-binding TF AceA induces the expression of the P-type ATPase $\operatorname{CrpA}$ for $\mathrm{Cu}$ detoxification (Antsotegi-Uskola et al., 2017; Wiemann et al., 2017; Cai et al., 2018; Yang et al., 2018).
Both AceA and CrpA are virulence determinants in the human pathogen A. fumigatus (Wiemann et al., 2017). Recently, Yang et al. illustrated that in the crossover pathogen $A$. flavus, deletion of P-type ATPases (A. flavus contains two, CrpA and CrpB) and the TF $A c e A$ resulted in significantly reduced virulence in mice but had no impact on virulence in maize seeds (Yang et al., 2018).

Fusarium graminearum species complex is the major causal agent of Fusarium head blight (FHB) or scab of wheat and barley, which is among the most important diseases of cereal crops worldwide (Bai and Shaner, 2004; Goswami and Kistler, 2004). Epidemics of FHB not only cause huge yield losses but also contaminate grains with harmful trichothecene mycotoxins, threatening the health of both humans and animals (Lombaert et al., 2003; Rasmussen et al., 2003; Tutelyan, 2004; Isebaert et al., 2005; Ji et al., 2014). Despite in-depth studies of copper homeostasis in human pathogens, information on the $\mathrm{Cu}$ detoxification machinery and the roles of this machinery in the virulence of plant pathogens is still limited, and thus we examined the possible existence and importance of $\mathrm{Cu}$-exporting mechanisms in the wheat pathogen F. graminearum.

The objectives of this study were to identify and characterize the major factors determining copper detoxification and their possible regulatory mechanisms in F. graminearum. Our results indicate that copper detoxification in the wheat pathogen F. graminearum relies mainly on $\mathrm{Cu}$ export similar to that in C. albicans and Aspergillus spp.

\section{MATERIALS AND METHODS}

\section{Strains, Culture Media, and Growth Conditions}

Fusarium graminearum strain PH-1 (NRRL 31084) (Cuomo et al., 2007) was used as the parental wild-type. To assess the mycelial growth, the wild-type strain $\mathrm{PH}-1$ and its corresponding mutants were grown on solidified FMM plates (Fusarium minimal medium) (Leslie and Summerell, 2007) amended under different $\mathrm{Cu}$ and/or menadione concentrations and incubated at $25^{\circ} \mathrm{C}$. Fungal biomass was compared by collecting mycelia from 3 -day-old liquid FMM culture incubated at $25^{\circ} \mathrm{C}$ in a shaker (180 rpm). For conidiation, five $5 \mathrm{~mm}$ mycelial plugs of the wild type strain $\mathrm{PH}-1$ and its mutants taken from the edge of a 3-dayold colony were inoculated in a $150 \mathrm{ml}$ triangular flask containing $50 \mathrm{ml}$ of CMC (carboxymethyl cellulose) medium and incubated at $25^{\circ} \mathrm{C}, 180 \mathrm{rpm}$ for 3 days in a shaker with light (Iida et al., 2008; Chen et al., 2018). The number of conidia in CMC medium was counted using a hemacytometer. Conidial germination rates were compared by re-suspending conidia in $2 \%$ sucrose solutions amended with or without $20 \mu \mathrm{M} \mathrm{Cu}$ at $25^{\circ} \mathrm{C}$ for 4 or $6 \mathrm{~h}$. Each experiment was carried out in triplicate and repeated for three times.

\section{Nucleic Acid Manipulation, PCR Primers, Mutant Generation, and Confirmation}

Using double-joint PCR strategy (Yu et al., 2004), deletion cassettes for each targeted gene were generated and transformed 
into the wild type $\mathrm{PH}-1$ by using polyethylene glycol (PEG)mediated protoplast transformation method (Proctor et al., 1995b). PCR primers used in this study were synthesized by Sangon Biotech (Shanghai, China) and are listed in Supplementary Table S1. Genes were deleted by creating constructs where $5^{\prime}$ and $3^{\prime}$ flanking regions were fused to the hygromycin gene $\mathrm{HPH}$ obtained from pBlueScript-hph or the G418 sulfate gene NEO from pBlueScript-neo. Solidified FMM supplemented with hygromycin (100 mg/L) or G418 sulfate (100 mg/L) was used to select transformants. PCR screenedpositive transformants were single spore isolated and stored in $15 \%$ glycerol at $-80^{\circ} \mathrm{C}$ for further experiments. To construct $\mathrm{FgAceA}^{\mathrm{c}}$ or FgCrpA ${ }^{\mathrm{c}}$, the full-length DNA of $\mathrm{FgAceA}$ or FgCrpA, including their promoter region (about $1.0 \mathrm{~kb}$ ), was cloned into the pYF11 plasmid (Bruno et al., 2004), and then the recombinant plasmid pYF11-FgAceA or pYF11-FgCrpA was transformed into its corresponding deletion mutant. Southern blot hybridization analysis of the mutants and its wild-type strain was performed using a detection starter kit II according to the manufacturer's instructions (Roche Diagnostics, Mannheim, Germany).

\section{Construction of Phylogenetic Tree}

Predicted protein sequences released in $\mathrm{FungiDB}^{1}$ were aligned with Clustal W (Thompson et al., 1994) and a phylogenetic tree was constructed with MEGA 5.10 software using a neighborjoining method (Kumar et al., 2008).

\section{RNA Extraction and Gene Expression Analysis}

Conidia $\left(10^{5}\right)$ of F. graminearum wild-type strain $\mathrm{PH}-1$ and its corresponding mutants were inoculated into $50 \mathrm{ml}$ liquid FMM in triplicates and cultured at $25^{\circ} \mathrm{C}, 180 \mathrm{rpm}$ for 3 days in a shaker. Mycelia was collected by filtering through sterile miracloth, washed with sterile water and then one half mycelia was grown under the same conditions with the other half shifted to a $\mathrm{Cu}$ treatment for several time points. Mycelia was then collected, washed with sterile water, frozen with liquid nitrogen and lyophilized for $24 \mathrm{~h}$. Total RNA of F. graminearum strains was extracted using TaKaRa RNAiso Plus (TaKaRa, Dalian, China) and cDNA was reverse transcripted using TaKaRa PrimeScript ${ }^{\mathrm{TM}}$ RT Master Mix (TaKaRa, Dalian, China). For quantitative Real-Time PCR (qRT-PCR) assays, expression of genes was determined with primer pairs listed in Supplementary Table S1. qRT-PCR amplifications were performed in a LightCycler ${ }^{\circledR} 96$ (Roche Molecular Systems, Inc.) using the TaKaRa SYBR ${ }^{\circledR}$ Premix Ex Taq ${ }^{\text {TM }}$ II (Tli RNaseH Plus) (TaKaRa, Dalian, China).

For RNA-seq analysis, $10^{5}$ conidia of the wild-type strain $\mathrm{PH}-1$ was inoculated into $50 \mathrm{ml}$ liquid FMM without $\mathrm{Cu}$ and cultured at $25^{\circ} \mathrm{C}$ with shaking $(180 \mathrm{rpm})$ for 3 days with a subsequent shift to $\mathrm{Cu}$-treated condition $(100 \mu \mathrm{M})$ for $1 \mathrm{~h}$ with triplicates as described above. RNA samples were prepared to perform digital transcriptome analysis by the RNA-seq approach (Shanghai Majorbio Bio-pharm Technology Co., Ltd.). The data

${ }^{1}$ http://fungidb.org/fungidb/ were analyzed on the free online platform of Majorbio I-Sanger Cloud Platform².

\section{Copper Quantification}

Conidia $\left(10^{5}\right)$ of F. graminearum wild-type strain $\mathrm{PH}-1$ and corresponding mutants were inoculated into $50 \mathrm{ml}$ liquid FMM in four replicates and cultured at $25^{\circ} \mathrm{C}, 180 \mathrm{rpm}$ for 3 days in a shaker. Subsequently, $\mathrm{Cu}$ was added to one half of the culture at a final concentration of 100 $\mu \mathrm{M}$ and fungi further cultured for $12 \mathrm{~h}$. Mycelia were then collected, washed by sterile water, and lyophilized. Quantification of $\mathrm{Cu}$ from $F$. graminearum strains was carried out according to the method previously described by Shanghai Microspectrum Chemical Technology Service Co., Ltd. (Yang et al., 2018).

\section{Virulence Tests}

Conidia of F. graminearum strains were harvested from 4-day-old CMC cultures, re-suspended in $0.01 \%$ (vol/vol) Tween 20 and adjusted to $1 \times 10^{5}$ conidia $/ \mathrm{ml}$. Virulence tests were performed using a single floret injection method as previously described ( $\mathrm{Wu}$ et al., 2005). Briefly, $10 \mu \mathrm{l}$ of conidial suspension $\left(1 \times 10^{5} / \mathrm{ml}\right)$ was injected into a single floret in the central spikelet of single flowering wheat heads of susceptible cultivar Zhenmai 10 at early anthesis with or without $3 \mathrm{~g} / \mathrm{L} \mathrm{Cu}$ fungicide (Nordox Cuprousoxide) spraying. Ten spikes were used for each strain. Infected spikelets in each inoculated wheat head were recorded and photographed 15 days after inoculation. The experiments were repeated three times.

\section{DON Production Assays}

Conidia $\left(10^{5}\right)$ of F. graminearum wild-type strain $\mathrm{PH}-1$ and corresponding mutants were inoculated into $30 \mathrm{ml}$ trichothecene biosynthesis inducing (TBI) liquid with or without $\mathrm{Cu}$ in three replicates and cultured at $28^{\circ} \mathrm{C}$ for 7 days. The filtrate and fungal mass were then collected separately, frozen with liquid nitrogen and lyophilized for $24 \mathrm{~h}$. The filtrate was redissolved in methanol and fungal mass was measured. The amount of DON in each sample was determined using a highperformance liquid chromatography-mass spectrometer/mass spectrometer (HPLC-MS/MS) system (Shimadzu 30A LC system coupled to a Triple Quad 6500 plus, Sciex, United States). Mass spectrometric parameters were according to a previously described method (Dong et al., 2016). The experiment was repeated two times.

\section{Statistical Analysis}

All data were presented as the means \pm standard deviation (SD). Statistical significance analysis was performed between the wild type parent $\mathrm{PH}-1$ and the deletion mutants with group $t$-tests with software package SPSS (version 13 for Windows, 2004). A $p$-value less than 0.05 was labeled as statistically significant.

\footnotetext{
${ }^{2}$ www.i-sanger.com
} 

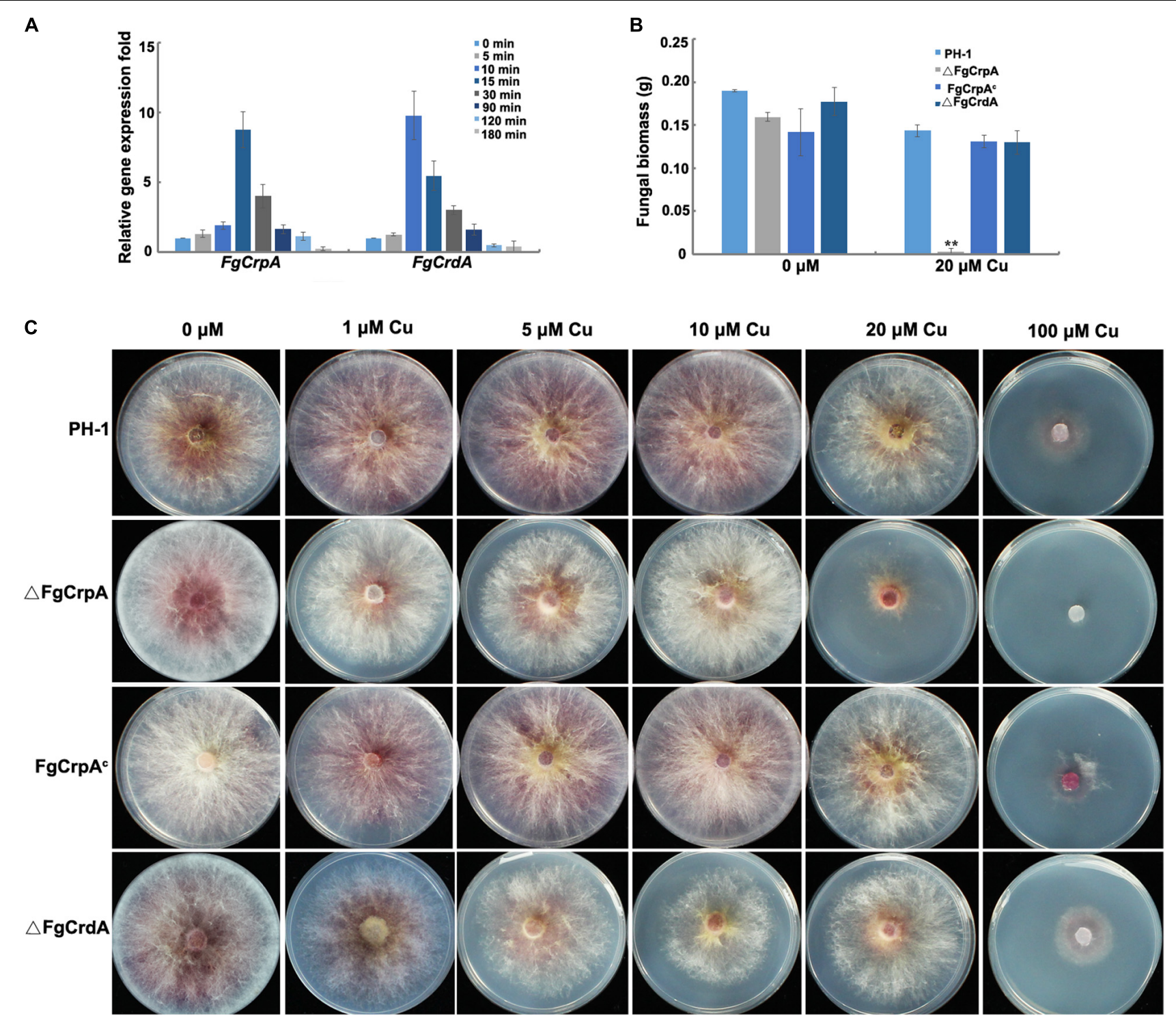

FIGURE 1 | FgCrpA and FgCrdA were induced by Cu treatment and their roles in Cu detoxification. (A) Expression of FgCrpA and FgCrdA in F. graminearum after $\mathrm{Cu}$ treatment analyzed by quantitative Real-time PCR assays. The wild-type strain $\mathrm{PH}-1$ was grown in liquid $\mathrm{FMM}\left(\mathrm{Cu}\right.$ free) for 3 days at $25^{\circ} \mathrm{C}$ and then collected, washed using sterile water and transferred to liquid FMM supplemented with $\mathrm{CuSO}_{4}$ at $100 \mu \mathrm{M}$ for different time courses. (B) Fungal growth was measured by determining the dry weight of the wild-type strain and mutants grown in $50 \mathrm{ml}$ liquid $\mathrm{FMM}$ amended with or without $\mathrm{CuSO}_{4}$ at $20 \mu \mathrm{M}$ in a shaker at $180 \mathrm{rpm}$ at $25^{\circ} \mathrm{C}$ for 3 days. Bars indicated means and error bars denote standard deviation from three repeated experiments. A $t$-test was performed to determine significant

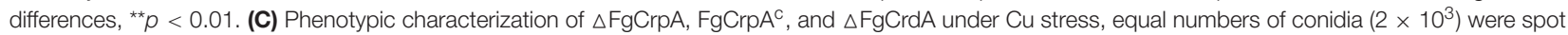
inoculated onto solidified FMM amended with $\mathrm{CuSO}_{4}$ at different concentrations indicated in the figure and cultured at $25^{\circ} \mathrm{C}$ for $3 d a y s$.

\section{RESULTS}

\section{Genes Involved in $\mathrm{Cu}$ Tolerance Revealed by RNA-Seq in $\boldsymbol{F}$. graminearum}

To gain a genomic perspective of the $\mathrm{Cu}$ tolerance mechanism in F. graminearum, we treated the wild-type strain $\mathrm{PH}$ 1 with or without $100 \mu \mathrm{M} \mathrm{CuSO}$ for $1 \mathrm{~h}$. Subsequently, RNA was extracted and prepared for RNA sequencing (RNA-Seq). All RNA-Seq raw data were deposited at the National Center for Biotechnology Information (NCBI)
Sequence Read Archive (SRA) ${ }^{3}$ under bioproject accession number PRJNA601796.

Overall, by comparing RNA-Seq data, we found that genes encoding putative $\mathrm{Cu}$ transporters were significantly differentially regulated following $100 \mu \mathrm{M} \mathrm{CuSO} 4$ treatment. In $F$. graminearum, six genes encoding putative $\mathrm{Cu}$-importing transporters, including the $S$. cerevisiae high-affinity $\mathrm{Cu}$ transporter Ctr1p ortholog FGRAMPH1_01G09843, the $\mathrm{S}$. cerevisiae high-affinity $\mathrm{Cu}$ transporter $\mathrm{Ctr} 2 \mathrm{p}$ homologs

${ }^{3}$ https://www.ncbi.nlm.nih.gov/sra 


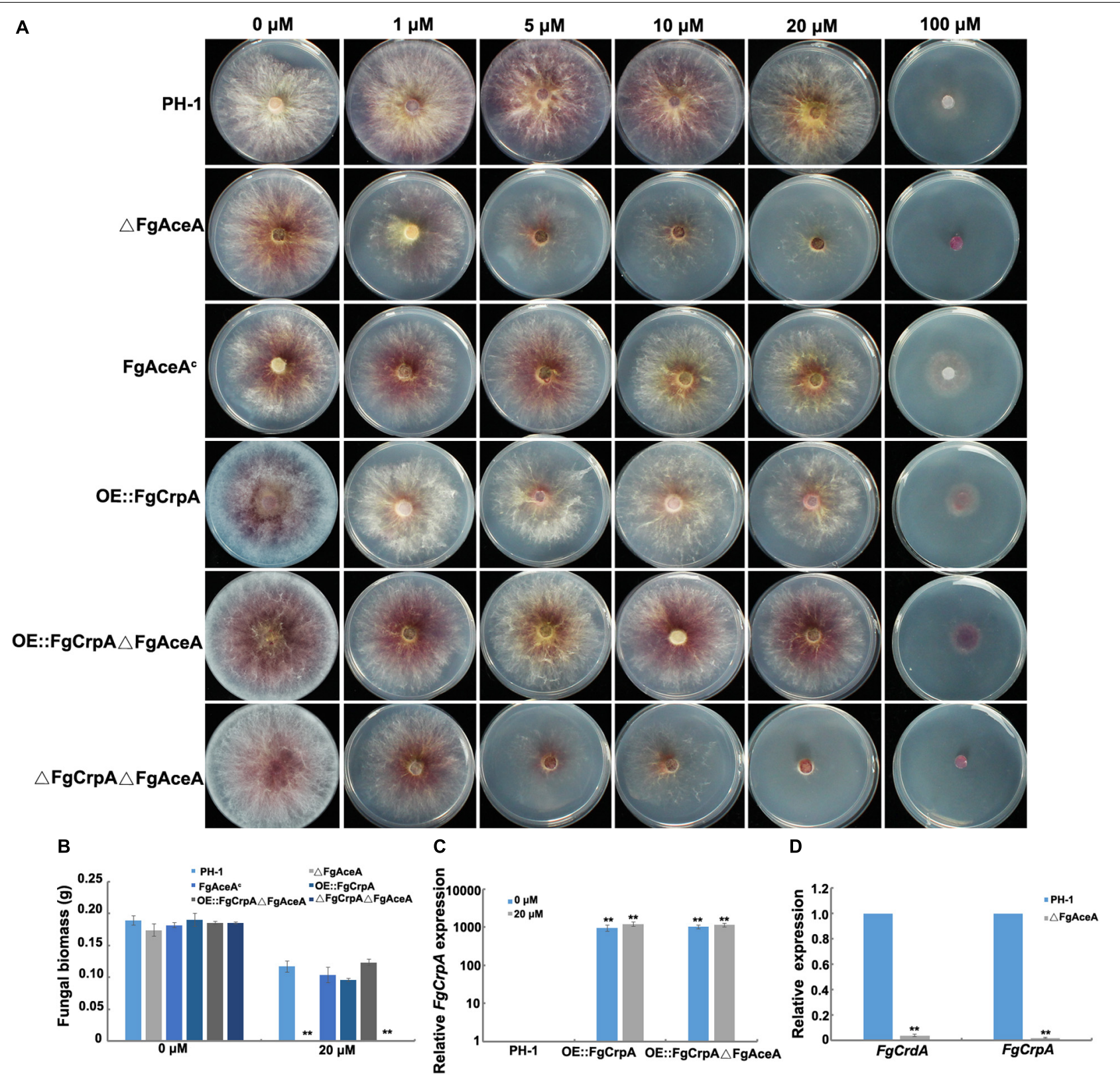

FIGURE 2 | Phenotypic analysis of $\triangle \mathrm{FgAceA}$ and its regulation on FgCrpA and FgCrdA. (A) Phenotypic characterization of the wild type strain PH-1 and mutants under $\mathrm{Cu}$ stress, equal numbers of conidia $\left(2 \times 10^{3}\right)$ were spot inoculated onto solidified $\mathrm{FMM}$ amended with $\mathrm{CuSO}_{4}$ at different concentrations indicated in the figure and cultured at $25^{\circ} \mathrm{C}$ for 3 days. (B) Fungal growth was measured by determining the dry weight of the wild type strain $\mathrm{PH}-1, \triangle \mathrm{FgAceA}, \mathrm{FgAceA}{ }^{\mathrm{C}}$, OE:FgCrpA, OE:FgCrpA $\triangle \mathrm{FgAceA}$, and $\triangle \mathrm{FgCrpA} \triangle \mathrm{FgAceA}$ grown in liquid $\mathrm{FMM}$ amended with or without $\mathrm{CuSO}_{4}$ at $20 \mu \mathrm{M}$ in a shaker at $180 \mathrm{rpm}$ at $25^{\circ} \mathrm{C}$ for 3 days. A $t$-test was performed to determine significant differences, ${ }^{\star *} p<0.01$. (C) Relative expression of FgCrpA in OE:FgCrpA or OE:FgCrpA $\triangle \mathrm{FgAceA}$ analyzed by qRT-PCR assays. (D) Relative expression of FgCrpA and FgCrdA in $\triangle$ FgAceA analyzed by qRT-PCR assays.

FGRAMPH1_01G19393 and FGRAMPH1_01G23855, the A. fumigatus $\mathrm{Cu}$ transporter CtrA1 orthologs FGRAMPH1_01G10595 and FGRAMPH1_01G13479, and the $S$. cerevisiae intracellular $\mathrm{Cu}$ ATPase Ccc2p ortholog FGRAMPH1_01G09315, were significantly downregulated (Supplementary Table S2). Furthermore, the C. albicans $\mathrm{Cu}$-exporting ATPase Crp1 ortholog FgCrpA (FGRAMPH1_01G10037) and the C. albicans MT Crd2 ortholog
FgCrdA (FGRAMPH1_01G09281) were significantly upregulated (Supplementary Table S2). Based on the observations from the RNA-Seq data, we next characterized the possible roles of the F. graminearum Crp1 ortholog (FgCrpA) and the Crd2 ortholog (FgCrdA) in $\mathrm{Cu}$ detoxification.

Previous studies have shown that the " $\mathrm{Cu}$ fist" DNA binding domain protein AceA was crucial in mediating $\mathrm{Cu}$ tolerance in Aspergillus spp. by activating the Cu-exporting 

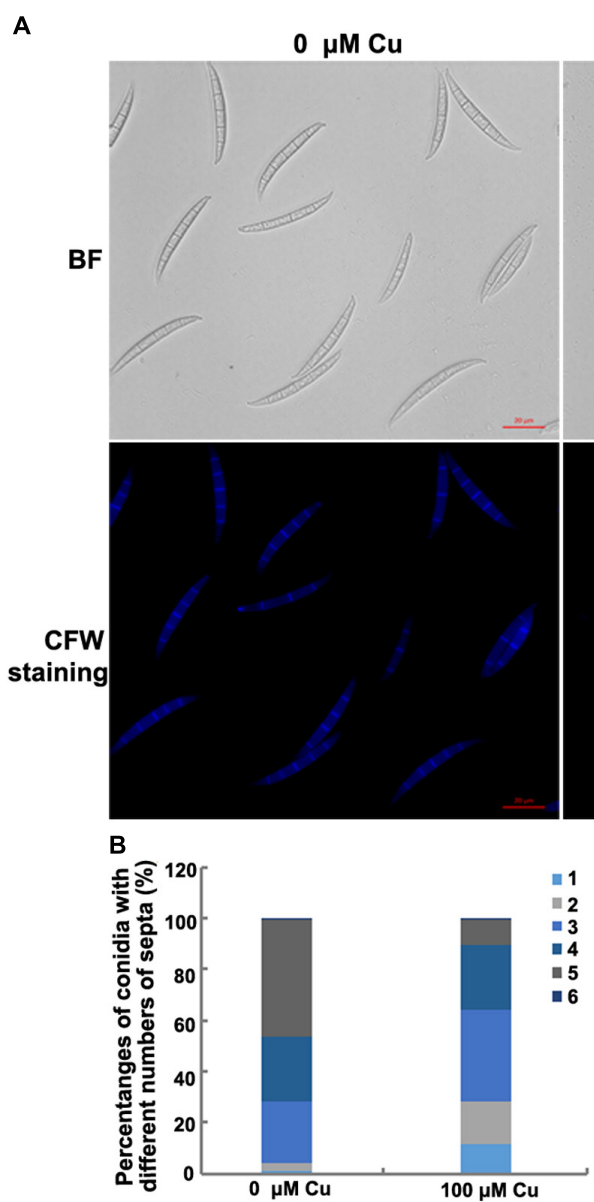

C

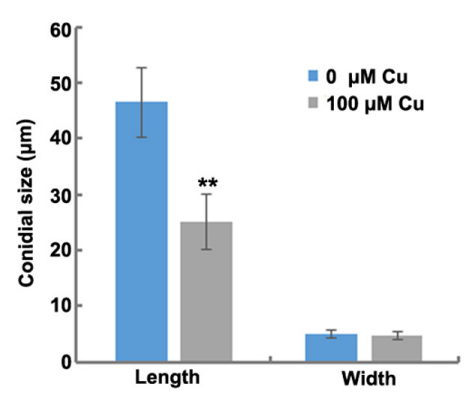

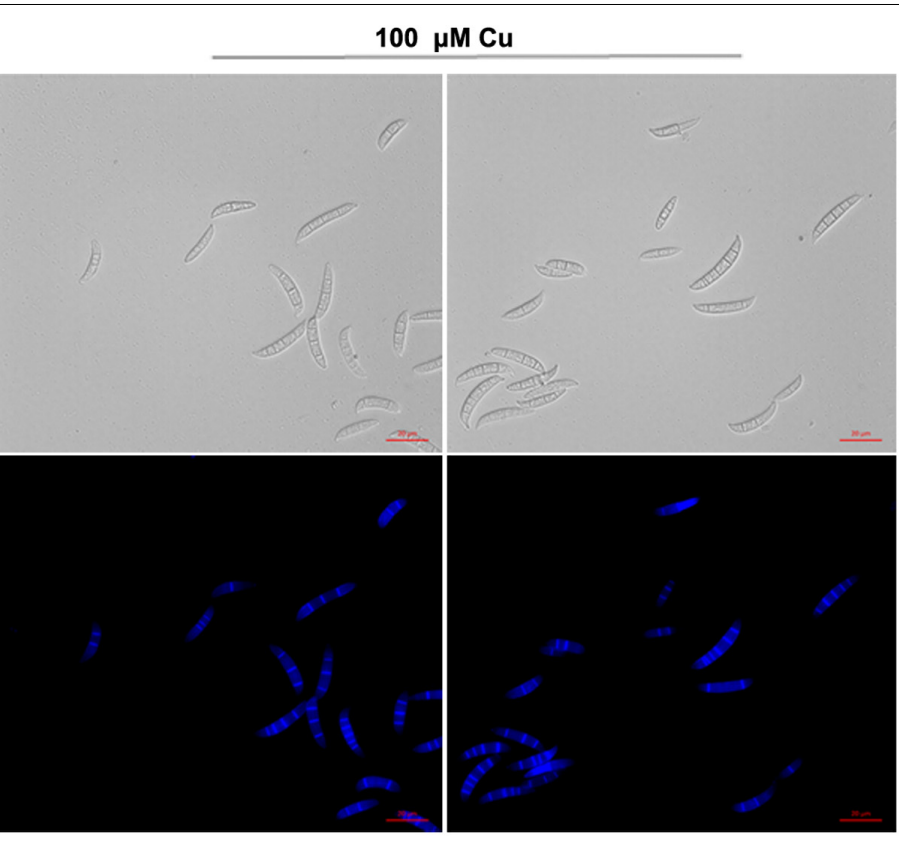

D

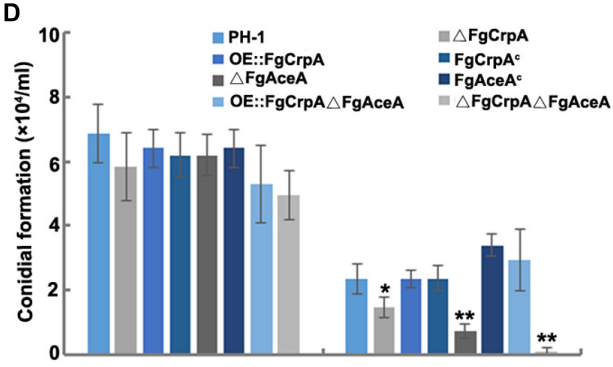

E

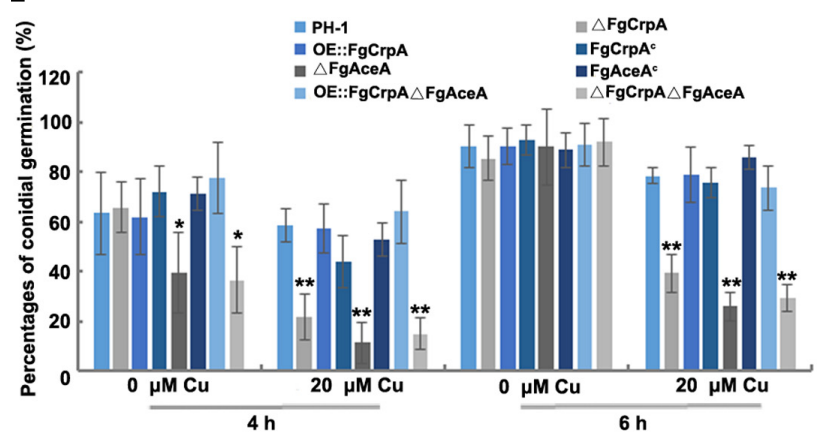

FIGURE 3 | Excess Cu treatment caused defects in conidial morphogenesis. Differences in F. graminearum conidial morphology (A), numbers of septa (B), and conidial size (C) between conidia treated and untreated with $\mathrm{Cu}$ at $100 \mu \mathrm{M}$. (D) Conidial formation was measured by inoculating five mycelial plugs taken from wild-type strain $\mathrm{PH}-1$ and its mutants in $50 \mathrm{ml}$ liquid $\mathrm{CMC}$ amended with or without $\mathrm{CuSO}_{4}$ at $20 \mu \mathrm{M}$ in a shaker with light at 180 rpm at $25^{\circ} \mathrm{C}$ for 3 days.

(E) Conidial germination was also compared between wild-type strain $\mathrm{PH}-1$ and its mutants by re-suspending the conidia in $10 \mathrm{ml}$ liquid FMM amended with or without $\mathrm{CuSO}_{4}$ at $20 \mu \mathrm{M}$ in a shaker with light at $180 \mathrm{rpm}$ at $25^{\circ} \mathrm{C}$ for $4 \mathrm{~h}$ and $6 \mathrm{~h}$. A $t$-test was performed to determine significant differences, ${ }^{*} p<0.05$, ${ }^{* *} p<0.01$.

transporters. The $S$. cerevisiae Ace1/YGL166W ortholog FgAceA (FGRAMPH1_01G09843) was identified using BlastP. Phylogenetic analysis showed that the $\mathrm{Cu}$-exporting ATPase FgCrpA, the MT FgCrdA, and the "Cu fist" DNA binding domain protein FgAceA shared high level of sequence identities with their orthologs in yeasts and Aspergillus spp. (Supplementary Figures S1A-C).

\section{Expression Patterns of FgCrpA and FgCrdA in Response to Cu Toxicity}

Quantitative real-time PCR (qRT-PCR) results showed that the expression of $F g C r p A$ and $F g C r d A$ in F. graminearum was induced rapidly by exposure to $100 \mu \mathrm{M} \mathrm{Cu}$. FgCrpA reached its maximal expression level after exposure to $\mathrm{Cu}$ treatment at $15 \mathrm{~min}$, followed by a decrease in expression (Figure 1A). FgCrdA 


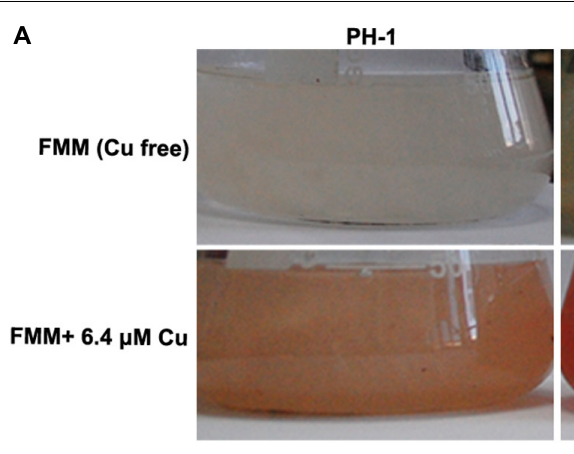

B

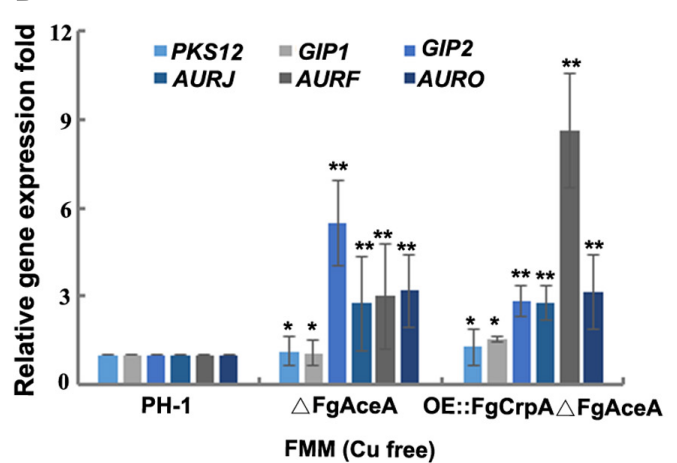

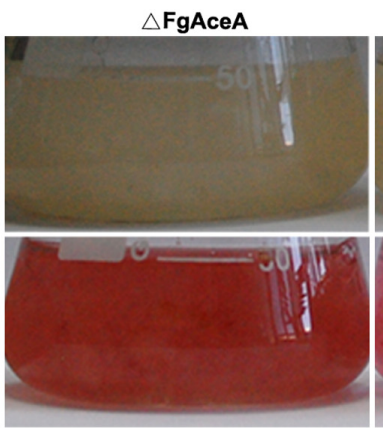

$\mathrm{OE}: \mathrm{FgCrpA} \triangle \mathrm{FgAceA}$

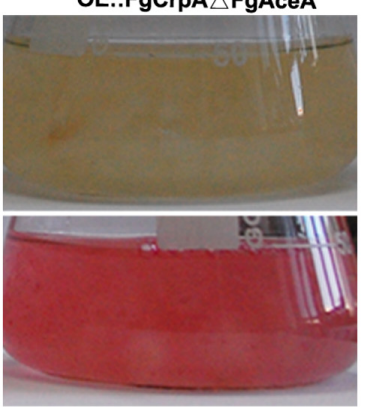

C

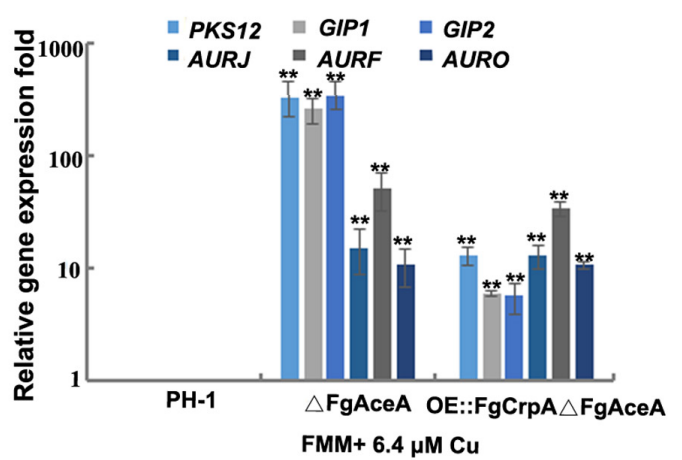

FIGURE 4 | Cu homeostasis affects aurofusarin accumulation in F. graminearum. (A) F. graminearum wild-type strain PH-1, $\triangle$ FgAceA, or OE:FgCrpA $\triangle$ FgAceA was cultured in $50 \mathrm{ml}$ liquid FMM with or without $5 \mu \mathrm{M}$ (trace element level) $\mathrm{Cu}$ in a shaker with light at $180 \mathrm{rpm}$ at $25^{\circ} \mathrm{C}$ for 4 days. Relative expression of six genes involved in aurofusarin biosynthesis (PKS12, GIP1, GIP2, AURJ, AURF, and AURO) in $\triangle \mathrm{FgAceA}$ or OE:FgCrpA $\triangle \mathrm{FgAceA}$ when grown in 50 ml liquid FMM with (B) or without $5 \mu \mathrm{M}$ (trace element level) (C) Cu in a shaker with light at $180 \mathrm{rpm}$ at $25^{\circ} \mathrm{C}$ for 4 days. A $t$-test was performed to determine significant differences, ${ }^{*} p<0.05$, ${ }^{* *} p<0.01$.

was also induced by $\mathrm{Cu}$ treatment, and its expression displayed a similar pattern as $\mathrm{FgCrpA}$; the maximal expression level was observed at $10 \mathrm{~min}$, followed by a decrease (Figure 1A).

\section{FgCrpA but Not FgCrdA Plays an Essential Role in $\mathrm{Cu}$ Tolerance in F. graminearum}

To determine the roles of $F g C r p A$ and $F g C r d A$ in $C u$ tolerance in F. graminearum, we generated the deletion mutants, $\triangle \mathrm{FgCrpA}$ and $\triangle$ FgCrdA, which were further confirmed by diagnostic PCR and Southern blot (Supplementary Figures S2, S3). Deletion of $\mathrm{FgCrpA}$ resulted in reduced tolerance to $\mathrm{Cu}$ concentrations of $20 \mu \mathrm{M}$ or higher (Figures 1B,C). However, no obvious difference in $\mathrm{Cu}$ tolerance was noticed between $\triangle \mathrm{FgCrdA}$ and the wild-type PH-1 (Figures 1B,C). Further, there were no additive $\mathrm{Cu}$ sensitivity effect observed in the $\triangle \mathrm{FgCrdA} \triangle \mathrm{FgCrpA}$ double mutant as compared with $\triangle \mathrm{FgCrpA}$ (data not shown). Transformation of the full-length $F g C r p A$ with its promoter region into $\triangle \mathrm{FgCrpA}$ rescued tolerance to $\mathrm{Cu}$ (Figures $\mathbf{1 B}, \mathbf{C}$ ).

\section{The Cu-Binding TF FgAceA Regulates FgCrpA During Cu Detoxification in F. graminearum}

The "Cu fist" DNA binding domain protein AceA has been identified in several Aspergillus spp. and proven to regulate the response to excess $\mathrm{Cu}$ by activating the $\mathrm{Cu}$-exporting transporters $\mathrm{CrpA}$ (and $\mathrm{CrpB}$ in A. flavus). In contrast, in Aspergillus the MT, CrdA, is not activated by AceA (Wiemann et al., 2017; Yang et al., 2018). To explore the roles of the F. graminearum AceA ortholog FgAceA in mediating $\mathrm{Cu}$ detoxification, an $\mathrm{FgAceA}$ full-length deletion strain, $\triangle \mathrm{FgAceA}$, was generated and confirmed by diagnostic PCR and Southern blot (Supplementary Figure S4). Deletion of FgAceA resulted in even more $\mathrm{Cu}$ sensitivity compared to $\triangle$ FgCrpA. $\triangle$ FgAceA barely grew on FMM supplemented with $5 \mu \mathrm{M} \mathrm{Cu}$. The double mutant $\triangle$ FgCrpA $\triangle$ FgAceA was more sensitive than $\triangle$ FgAceA when the FMM was treated with $20 \mu \mathrm{M} \mathrm{Cu}$ (Figures 2A,B). Complementation with the full-length FgAceA driven by its native promoter restored $\mathrm{Cu}$ tolerance to that of wild type (Figures 2A,B).

To investigate the regulatory mechanism of $F g A c e A$ in F. graminearum $\mathrm{Cu}$ tolerance, we generated an overexpression strain of $F g C r p A$ (OE:FgCrpA) by replacing its native promoter with the constitutive promoter $g p d A$ from $A$. nidulans in both the wild-type strain PH-1 and $\triangle$ FgAceA. OE:FgCrpA strains were screened by diagnostic PCR and further confirmed by Southern blot (Supplementary Figure S5) and qRTPCR. The transcriptional level of $F g C r p A$ was significantly upregulated with or without $\mathrm{Cu}$ treatment (Figure 2C). OE:FgCrpA exhibited a restored $\mathrm{Cu}$ tolerance in the $\triangle$ FgAceA background (Figures 2A,B), however, OE::FgCrpA in the 


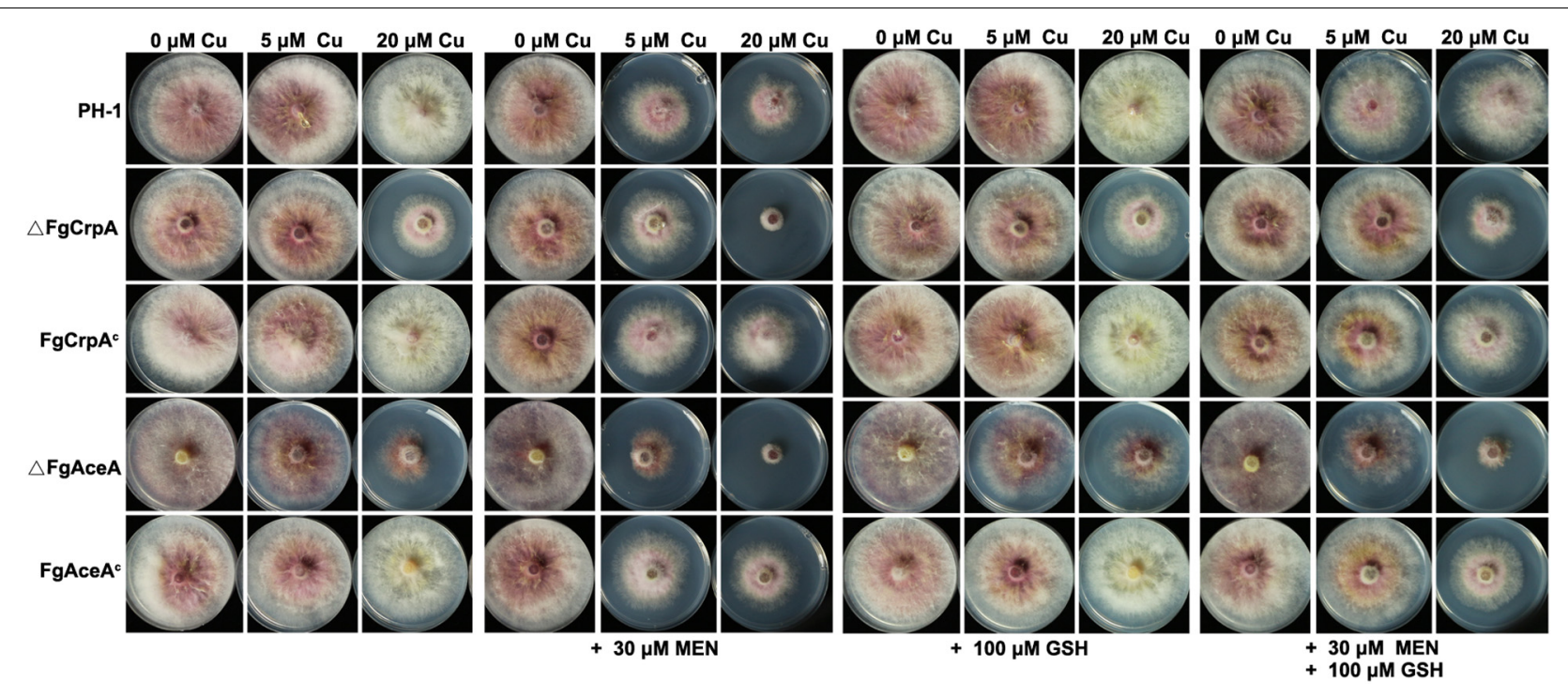

FIGURE 5 | Reduced Cu tolerance caused by FgAceA or FgCrpA deletion aggravates ROS sensitivity in F. graminearum. Growth of F. graminearum strains on Cu-containing FMM media added with supplements (menadione, MEN and/or L-glutathione, GSH) at various concentrations as indicated at $25^{\circ} \mathrm{C}$ for 4 days.

wild-type strain $\mathrm{PH}-1$ did not exhibit an increased $\mathrm{Cu}$ tolerance (Figure 2A). Deletion of FgAceA ameliorated the induced $F g C r p A$ and $F g C r d A$ expression after excess $\mathrm{Cu}$ exposure (Figure 2D).

\section{Deletion of FgAceA and FgCrpA Caused Defects in Conidial Formation and Germination Under Excess $\mathrm{Cu}$ Conditions in F. graminearum}

F. graminearum produced atypical conidia with shorter sizes and fewer septa when cultured under excess $\mathrm{Cu}$ conditions (Figures 3A-C). Conidial formation and germination were significantly reduced in $\triangle$ FgCrpA $(p<0.05), \triangle$ FgAceA $(p<0.01)$ and $\Delta$ FgCrpA $\Delta$ FgAceA $(p<0.01)$ under 100 $\mu \mathrm{M} \mathrm{Cu}$ treatment (Figures 3D,E). The double deletion mutant $\Delta$ FgCrpA $\Delta$ FgAceA was more sensitive to $\mathrm{Cu}$ as reflected by greater impairment of both conidial formation and germination compared with both single deletion mutants (Figures 3D,E). Noticeably, deletion of FgAceA caused reduced conidial germination even without excess $\mathrm{Cu}$ challenge at $4 \mathrm{~h}$ but was restored to wild-type level when the germination time was extended to $6 \mathrm{~h}$ (Figure 3E).

\section{Altered FgAceA Expression Affects Aurofusarin Accumulation in F. graminearum}

One immediately observed phenotype of $\Delta$ FgAceA grown in high $\mathrm{Cu}$ medium was an intense red pigmentation (Figure 4A). We hypothesized that this pigment was the known secondary metabolite aurofusarin and confirmed this likelihood by the dense red color produced. qRT-PCR results which showed that in liquid FMM with or without $\mathrm{Cu}$, the expression of six genes (PKS12, GIP1, GIP2, AURJ, AURF, and AURO) involved in aurofusarin biosynthesis increased dramatically in $\triangle F g A c e A$, and trace amounts of $\mathrm{Cu}$ stimulated the expression of these six genes (Figure 4B).

\section{Reduced Cu Tolerance Caused by FgAceA or FgCrpA Deletion Aggravates ROS Sensitivity in F. graminearum}

Previous studies have shown that excess $\mathrm{Cu}$ could generate reactive oxygen species such as hydroxyl radicals (Ding et al., 2013, 2014). To test whether $\mathrm{Cu}$ detoxification was involved in mediating reactive oxygen intermediates (ROI) stress in F. graminearum, wild type strain $\mathrm{PH}-1, \Delta \mathrm{FgCrpA}, \triangle \mathrm{FgAceA}$ and their complementary strains were challenged with excess $\mathrm{Cu}$ and menadione. Results showed that $30 \mu \mathrm{M}$ menadione and increasing $\mathrm{Cu}$ had inhibitory effects on all the F. graminearum strains tested. Reduced $\mathrm{Cu}$ tolerance caused by deletion of FgAceA or FgCrpA aggravates ROS sensitivity as demonstrated by the reduced growth of $\triangle \mathrm{FgCrpA}$ and $\triangle \mathrm{FgAceA}$ when grown on $\mathrm{Cu}$ containing FMM media supplemented with $30 \mu \mathrm{M}$ menadione (Figure 5). Moreover, the reductant L-glutathione (GSH) could relieved the severe phenotype of all strains (Figure 5), suggesting that compromises in the $\mathrm{Cu}$ detoxification pathway aggravates ROI toxicity.

\section{Reduced Cu Tolerance Hinders Virulence and DON Biosynthesis in F. graminearum}

Pathogenicity assay results showed that the ability to invade untreated flowering wheat heads was not impaired by the single or double deletion of FgAceA and FgCrpA, as shown in Figure 6A (upper panel). However, when inoculated onto $\mathrm{Cu}$ fungicide-treated flowering wheat heads, $\triangle \mathrm{FgCrpA}$, $\Delta$ FgAceA, and $\Delta$ FgCrpA $\Delta$ FgAceA could not successfully colonize the inoculated spikelet, thus completely losing their 


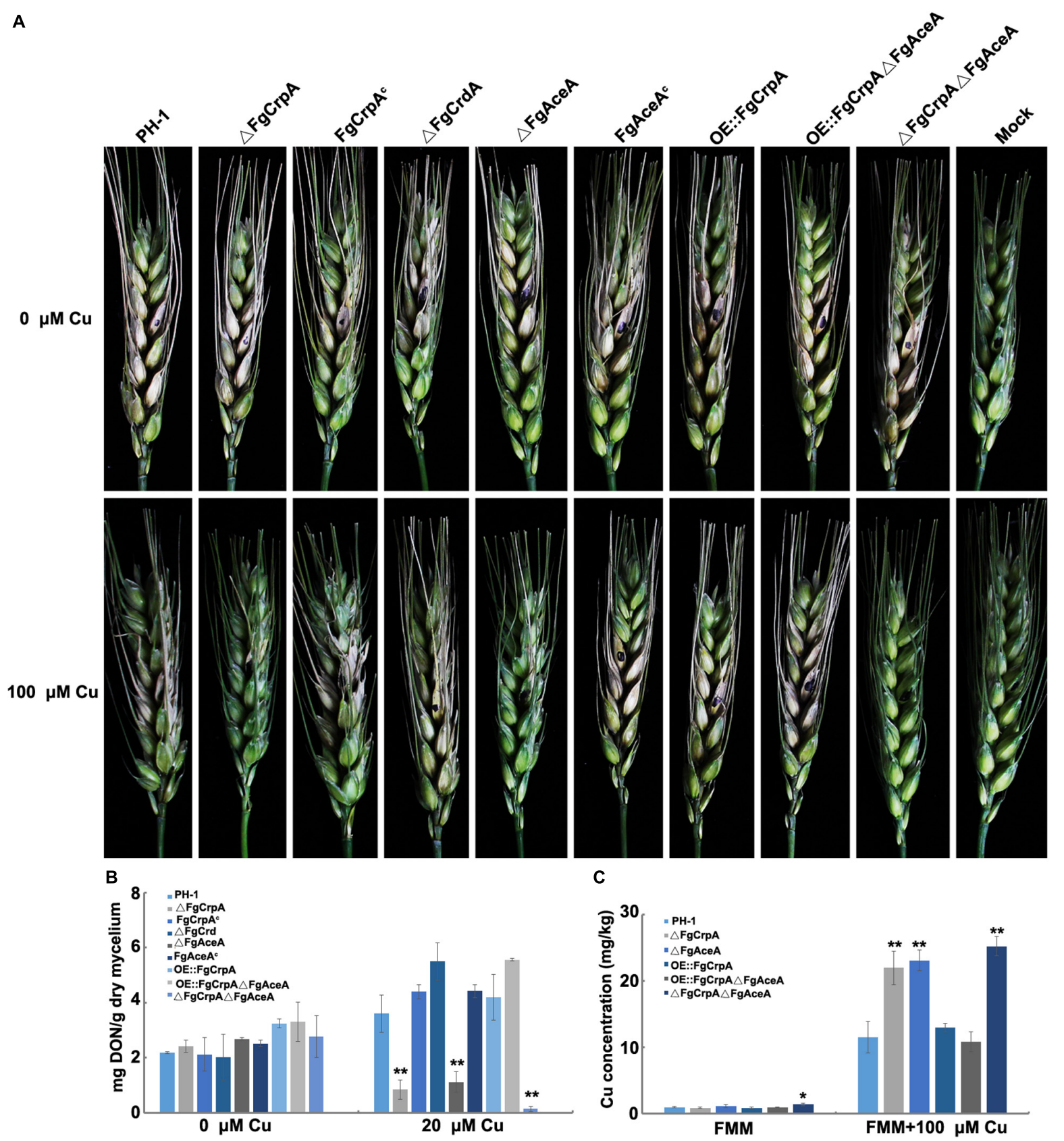

FIGURE 6 | Comparisons of virulence, DON accumulation and Cu concentration between wild-type strain PH-1 and its mutants. (A) Difference in virulence of F. graminearum strains inoculated on Cu treated and untreated flowering wheat heads. (B) Difference in the ability of DON accumulation virulence of $F$. graminearum strains inoculated in $30 \mathrm{ml}$ liquid TBI (inoculum: $10^{5}$ spores) with or without $20 \mu \mathrm{M} \mathrm{Cu}$ and cultured at $28^{\circ} \mathrm{C}$ for 7 days. (C) Difference in Cu concentration in mycelium collected from the wild-type strain $\mathrm{PH}-1$ and its mutants treated or untreated with $100 \mu \mathrm{M}$ Cu for $12 \mathrm{~h}$. A t-test was performed to determine significant differences, ${ }^{\star} p<0.05,{ }^{\star *} p<0.01$.

aggressiveness (Figure 6A, lower panel). Reintroduction of FgCrpA or FgAceA to their respective deletion mutants and overexpression of $\mathrm{FgCrpA}$ in $\triangle \mathrm{FgAceA}$ rescued their lost virulence on $\mathrm{Cu}$ fungicide-treated flowering wheat heads (Figure 6A, lower panel). Deletion of $\mathrm{FgCrdA}$ did not result in visible changes in virulence in $F$. graminearum regardless of $\mathrm{Cu}$ treatment (Figure 6A).
The toxic secondary metabolite deoxynivalenol (DON) produced by $F$. graminearum is a known virulence factor during plant infection (Proctor et al., 1995a; Ilgen et al., 2008). The reduced $\mathrm{Cu}$ tolerance caused by disruption of FgAceA and/or FgCrpA also affected DON accumulation. As shown in Figure $6 \mathbf{B}, \Delta \mathrm{FgCrpA}, \triangle \mathrm{FgAceA}$, and $\Delta \mathrm{FgCrpA} \Delta \mathrm{FgAceA}$ produced significantly $(p<0.01)$ lower amounts of DON under 
$20 \mu \mathrm{M} \mathrm{Cu}$ treatment than did the wild-type strain PH-1. Reintroduction of $\mathrm{FgCrpA}$ or $\mathrm{FgAceA}$ to their respective deletion mutants and overexpression of $\mathrm{FgCrpA}$ in $\triangle \mathrm{FgAceA}$ rescued DON biosynthesis. Deletion of $\mathrm{FgCrdA}$ did not result in changes in DON biosynthesis in F. graminearum. When treated with 20 $\mu \mathrm{M} \mathrm{Cu}$, the $F$. graminearum wild-type strain $\mathrm{PH}-1$ accumulated more DON than the untreated TBI liquid (Figure 6B).

Next, we determined the $\mathrm{Cu}$ concentration in the mycelium and compared the differences between the mutants and their wild-type strain $\mathrm{PH}-1$. The results showed that when cultured in FMM liquid (trace level of $\mathrm{Cu}$ ), only $\Delta$ FgCrpA $\Delta$ FgAceA showed significantly $(p<0.05)$ increased cellular accumulation of $\mathrm{Cu}$ compared to the wild-type strain $\mathrm{PH}-1$ (Figure 6C). However, when cultured in excess $\mathrm{Cu}$ (FMM liquid amended with $100 \mu \mathrm{M})$, the $\mathrm{Cu}$ concentration in $\Delta \mathrm{FgCrpA}, \Delta \mathrm{FgAceA}$, and $\Delta$ FgCrpA $\Delta$ FgAceA increased significantly $(p<0.01)$ compared with the wild-type strain $\mathrm{PH}-1$ or the complementary strain. Additionally, the overexpression of $F g C r p A$ in $\triangle$ FgAceA rescued the $\mathrm{Cu}$ export deficiency of the mutant, and the $\mathrm{Cu}$ level decreased to that of the wild-type (Figure 6C).

\section{DISCUSSION}

Accumulating evidence suggests that during infection, invading fungal pathogens will be confronted with elevated levels of essential trace nutrients harnessed by the host, collectively known as "nutritional immunity" (Ballou and Wilson, 2016). Moreover, fungal pathogens have also evolved an accurate mechanism to balance the fine lines between the essentiality and toxicity of these essential trace nutrients to survive and colonize within the infected host. For example, all living organisms maintain $\mathrm{Cu}$ homeostasis by regulating the balance between $\mathrm{Cu}$ uptake, utilization and detoxification (Ding et al., 2014; Ballou and Wilson, 2016). In this study, we characterized the copper tolerance determinants in the important wheat head blight fungus $F$. graminearum. Overall, our study showed that F. graminearum $\mathrm{Cu}$ resistance is mainly mediated by the ATPbased export system and not by a Cu MT.

The P-type ATPase FgCrpA and the MT FgCrdA were both found to be significantly upregulated when treated with excess $\mathrm{Cu}$ in RNA-Seq analysis and further verified by qRT-PCR. This was consistent with observation in Aspergillus spp. Cu-induced $\operatorname{Crp} A$ and $\operatorname{Crp} B$ expression was confirmed in $A$. flavus by using Northern Blot analysis (Yang et al., 2018). In A. nidulans, $\mathrm{Cu}-$ induced CrpA and CrdA protein expression was confirmed by Western blot (Antsotegi-Uskola et al., 2017). As Crp and Crd shared conserved expression pattern and functions in response to $\mathrm{Cu}$ toxicity, we expected protein expression of FgCrpA and FgCrdA to be induced in F. graminearum. The results of gene disruption assays indicated crucial roles of $F g C r p A$ but not $\mathrm{FgCrdA}$ in mediating $\mathrm{Cu}$ resistance in F. graminearum, indicating that $\mathrm{Cu}$ efflux is the predominant mechanism of $\mathrm{Cu}$ detoxification rather than MT-mediated $\mathrm{Cu}$ buffering. Together with previous studies carried out in other fungi, including $C$. albicans and Aspergillus spp. (A. fumigatus, A. flavus, A. nidulans), these results indicated that ATP-based efflux, as the principal $\mathrm{Cu}$ detoxification mechanism, is not unique and likely a well-conserved feature among filamentous fungal species (Antsotegi-Uskola et al., 2017; Wiemann et al., 2017; Cai et al., 2018; Yang et al., 2018).

Apart from Cu efflux, MT-related buffering also plays critical roles in $\mathrm{Cu}$ detoxification for some fungi. MTs function as $\mathrm{Cu}$ storage proteins by chelating excess $\mathrm{Cu}$ in yeast species. In $S$. cerevisiae, cells are protected from excess $\mathrm{Cu}$ by activating the MT-encoding genes cup 1 and crs5, and surplus $\mathrm{Cu}$ is then coordinated (Ecker et al., 1986; Culotta et al., 1994; Palacios et al., 2011; Thiele, 2015). In the human fungal pathogen Cryptococcus neoformans, the $\mathrm{Cu}$ metallo-regulatory TF Cuf1 activates the $\mathrm{Cu}$-buffering MT-encoding genes $\mathrm{Mt1}$ and $\mathrm{Mt} 2$ for $\mathrm{Cu}$ detoxification (Ding et al., 2013; Garcia-Santamarina et al., 2017). In the soil organism F. oxysporum, the Cu metallothionein Mt1 has been shown to be involved in mediating resistance to metal toxicity and virulence (Lorenzo-Gutiérrez et al., 2019). Despite the important roles of MTs in Cu detoxification in yeast species and F. oxysporum, their homologs in other filamentous fungi, including A. flavus, A. fumigatus, and A. nidulans, have been reported to be not particularly important. Deletion of $\mathrm{CrdA}$ caused no significant differences in $\mathrm{Cu}$ tolerance in these Aspergillus spp. In F. graminearum, although $\mathrm{FgCrdA}$ has been identified to respond to excess $\mathrm{Cu}$ treatment, its disruption $\mathrm{did}$ not alter $\mathrm{Cu}$ tolerance in $\triangle \mathrm{FgCrdA}$, the exact role of $\mathrm{FgCrdA}$ in $\mathrm{Cu}$ detoxification remains to be explored. Alternatively, $\mathrm{Cu}$ binding secondary metabolites - possibly filling a MT-like function - are hypothesized to be involved in $\mathrm{Cu}$ homeostasis in filamentous fungi (Raffa et al., 2019).

$\mathrm{Cu}$ homeostasis is tightly regulated in $F$. graminearum by the $\mathrm{Cu}$-responsive TF FgAceA. We showed that deletion of FgAceA resulted in reduced $\mathrm{Cu}$ tolerance and ceased the induction of FgCrpA expression by excess Cu loading, suggesting that $F g C r p A$ is transcriptionally expressed in an FgAceA-dependent manner under excess $\mathrm{Cu}$ stress. Overexpression of $\mathrm{FgCrpA}$ in $\triangle \mathrm{FgAceA}$ could compensate for the reduced $\mathrm{Cu}$ tolerance, indicating that FgAceA mediates $\mathrm{Cu}$ homeostasis by regulating the expression of the copper exporting P-type ATPase FgCrpA. In S. cerevisiae, Ace1 functions by recognizing and binding to the $\mathrm{Cu}$-dependent responsive element $\mathrm{ACE}$ in the promoter regions of its target MT-encoding genes cup 1 and crs 5 and the superoxide dismutase gene sod1. ACE contains the core sequence $5^{\prime}$-HTHXXGCTGD$3^{\prime}(\mathrm{D}=\mathrm{A}, \mathrm{G}$, or $\mathrm{T} ; \mathrm{H}=\mathrm{A}, \mathrm{C}$ or $\mathrm{T}$; and $\mathrm{X}=$ any residue). The promoter region of $\mathrm{FgCrpA}$ contains one core $\mathrm{ACE}$ sequence (TTATGCTGT), indicating a potential FgAceA binding domain.

$\mathrm{Cu}$ could not be properly transported out of the cell and was accumulated in mutants lacking $\mathrm{FgAceA}$ or FgCrpA (Figure 6C); thus, these mutants displayed compromised phenotypes when challenged with excess $\mathrm{Cu}$, as expected. Conidial formation and germination as well as DON biosynthesis and the ability to colonize flowering wheat heads were impaired in $\triangle$ FgAceA and $\triangle$ FgCrpA when treated with high levels of $\mathrm{Cu}$. We found that $\mathrm{FgAceA}$ negatively regulates aurofusarin biosynthesis. In both liquid FMM medium with and without trace amounts of $\mathrm{Cu}$, the accumulation of the red pigment aurofusarin increased significantly in $\triangle$ FgAceA compared with its wildtype parent. Six genes involved in aurofusarin biosynthesis were significantly upregulated in $\triangle$ FgAceA and OE:FgCrpA $\triangle$ FgAceA 
(Figures 4B,C). Overexpression of FgCrpA in $\triangle$ FgAceA did not restore the overproduction of aurofusarin, suggesting that $\mathrm{FgAce} A$ has additional roles in negatively regulating secondary metabolism and bypassing the mediation of $\mathrm{Cu}$ tolerance.

FgAceA and/or FgCrpA deletion mutants could not colonize on $\mathrm{Cu}$ fungicide-treated flowering wheat heads. Several reasons could account for the virulence loss of the mutants. First, it could not be separated from the defects in conidial germination and reduced aerial hyphae under excess $\mathrm{Cu}$ treatment. Second, ROS such as hydroxyl radicals generated with excess $\mathrm{Cu}$ or plant oxidative burst could also be responsible for the unsuccessful colonization of the mutants. Additionally, DON has been identified as a crucial virulence factor and plays an important role in aggressiveness of the fungus among the spikelets of the infected wheat head, the decreased DON production in the mutants challenged with excess $\mathrm{Cu}$ may also account for the loss of full virulence.

In summary, we elucidated the copper tolerance mechanism in the important wheat head blight fungus F. graminearum, which is mainly mediated by the ATP-based extrusion system. The P-type ATPase $F g C r p A$ is transcriptionally regulated by the $\mathrm{Cu}$-fist TF $\mathrm{FgAceA}$, and both are crucial for $\mathrm{Cu}$ tolerance.

\section{DATA AVAILABILITY STATEMENT}

The datasets presented in this study can be found in online repositories. The names of the repository/repositories and accession number(s) can be found in the article/ Supplementary Material.

\section{AUTHOR CONTRIBUTIONS}

$\mathrm{XL}$ and YJ carried out the experimentation of this work. JS and NK conceived the experiments and wrote the manuscript. DH, $\mathrm{XF}$, and JX helped create strains for this research. Y-WL helped revise this manuscript. All authors contributed to the article and approved the submitted version.

\section{REFERENCES}

Antsotegi-Uskola, M., Markina-Iñarrairaegui, A., and Ugalde, U. (2017). Copper resistance in Aspergillus nidulans relies on the P-type ATPase $\operatorname{CrpA}$, regulated by the transcription factor AceA. Front. Microbiol. 8:912. doi: 10.3389/fmicb. 2017.00912

Babula, P., Masarik, M., Adam, V., Eckschlager, T., Stiborova, M., Trnkova, L., et al. (2012). Mammalian metallothioneins: properties and functions. Metallomics 4, 739-750. doi: 10.1039/c2mt20081c

Bai, G., and Shaner, G. (2004). Management and resistance in wheat and barley to Fusarium head blight. Annu. Rev. Phytopathol. 42, 135-161. doi: 10.1146/ annurev.phyto.42.040803.140340

Ballou, E. R., and Wilson, D. (2016). The roles of zinc and copper sensing in fungal pathogenesis. Curr. Opin. Microbiol. 32, 128-134. doi: 10.1016/j.mib.2016.05. 013

Besold, A. N., Culbertson, E. M., and Culotta, V. C. (2016). The Yin and Yang of copper during infection. J. Biol. Inorg. Chem. 21, 137-144. doi: 10.1007/s00775016-1335-1

\section{FUNDING}

This work was supported by the National Key R\&D Program of China (2018YFE0206000), National Natural Science Fund of China (31772118, 31701748, and 31601594), Natural Science Fund of Jiangsu Province (BK20181246), Jiangsu Agriculture Science and Technology Innovation Fund (CX(19)3004), Special Fund for Risk Assessment of China (GJFP2019002), and Shanghai Agriculture Applied Technology Development Program (2019-02-08-00-12-F01148).

\section{ACKNOWLEDGMENTS}

We thank the native English-speaking scientists of American Journal Experts (Durham, NC, United States) for editing our manuscript.

\section{SUPPLEMENTARY MATERIAL}

The Supplementary Material for this article can be found online at: https://www.frontiersin.org/articles/10.3389/fmicb. 2020.01392/full\#supplementary-material

FIGURE S1 | Bioinformatic analysis of putative Cu detoxification determinants.

FIGURE S2 | Diagram for generation and verification for $\triangle$ FgAceA by Southern blotting analysis.

FIGURE S3 | Diagram for generation and verification for $\triangle$ FgCrpA by Southern blotting analysis.

FIGURE S4 | Diagram for generation and verification for $\triangle$ FgCrdA by Southern blotting analysis.

FIGURE S5 | Diagram for generation and verification for OE:FgCrpA by Southern blotting analysis.

TABLE S1 | PCR primers used in this study.

TABLE S2 | Expression of genes encoding putative copper-binding proteins revealed by RNA-seq.

Bruno, K. S., Tenjo, F., Li, L., Hamer, J. E., and Xu, J. R. (2004). Cellular localization and role of kinase activity of PMK1 in Magnaporthe grisea. Eukaryot. Cell 3, 1525-1532. doi: 10.1128/ec.3.6.1525-1532.2004

Cai, Z., Du, W., Zhang, Z., Guan, L., Zeng, Q., Chai, Y., et al. (2018). The Aspergillus fumigatus transcription factor AceA is involved not only in $\mathrm{Cu}$ but also in $\mathrm{Zn}$ detoxification through regulating transporters $\operatorname{CrpA}$ and $\mathrm{ZrcA}$. Cell. Microbiol. 20:e12864. doi: 10.1111/cmi.12864

Chen, L., Tong, Q., Zhang, C., and Ding, K. (2018). The transcription factor $\mathrm{FgCrz} 1 \mathrm{~A}$ is essential for fungal development, virulence, deoxynivalenol biosynthesis and stress responses in Fusarium graminearum. Curr. Genet. 65, 1-14. doi: 10.1007/s00294-018-0853-5

Culotta, V. C., Howard, W. R., and Liu, X. F. (1994). CRS5 encodes a metallothionein-like protein in Saccharomyces cerevisiae. J. Biol. Chem. 269, 25295-25302.

Cuomo, C. A., Güldener, U., Xu, J. R., Trail, F., Turgeon, B. G., Di Pietro, A., et al. (2007). The Fusarium graminearum genome reveals a link between localized polymorphism and pathogen specialization. Science 317, 1400-1402. doi: 10 . 1126/science. 1143708 
Cyert, M. S., and Philpott, C. C. (2013). Regulation of cation balance in Saccharomyces cerevisiae. Genetics 193, 677-713. doi: 10.1534/genetics.112. 147207

Dalecki, A. G., Crawford, C. L., and Wolschendorf, F. (2017). Copper and antibiotics: discovery, modes of action, and opportunities for medicinal applications. Adv. Microb. Physiol. 70, 193-260. doi: 10.1016/bs.ampbs.2017. 01.007

Ding, C., Festa, R. A., Chen, Y. L., Espart, A., Palacios, Ò, Espín, J., et al. (2013). Cryptococcus neoformans copper detoxification machinery is critical for fungal virulence. Cell Host Microbe 13, 265-276. doi: 10.1016/j.chom.2013.02.002

Ding, C., Festa, R. A., Sun, T. S., and Wang, Z. Y. (2014). Iron and copper as virulence modulators in human fungal pathogens. Mol. Microbiol. 93, 10-23. doi: $10.1111 / \mathrm{mmi} .12653$

Ding, C., Yin, J., Tovar, E. M. M., Fitzpatrick, D. A., Higgins, D. G., and Thiele, D. J. (2011). The copper regulon of the human fungal pathogen Cryptococcus neoformans H99. Mol. Microbiol. 81, 1560-1576. doi: 10.1111/j.1365-2958.2011. 07794.x

Dollwet, H. H. A., and Sorenson, J. R. J. (1985). Historic uses of copper compounds in medicine. J Trace Elem. Med. Bio. 2, 80-87. doi: 10.1093/eurpub/11.2.238

Dong, F., Qiu, J., Xu, J., Yu, M., Wang, S., Sun, Y., et al. (2016). Effect of environmental factors on Fusarium population and associated trichothecenes in wheat grain grown in Jiangsu province, China. Int. J. Food Microbiol. 230, 58-63. doi: 10.1016/j.ijfoodmicro.2016.04.020

Ecker, D. J., Butt, T. R., Sternberg, E. J., Neeper, M. P., Debouck, C., Gorman, J. A., et al. (1986). Yeast metallothionein function in metal ion detoxification. J. Biol. Chem. 261, 16895-16900.

Festa, R. A., Helsel, M. E., Franz, K. J., and Thiele, D. J. (2014). Exploiting innate immune cell activation of a copper-dependent antimicrobial agent during infection. Chem. Biol. 21, 977-987. doi: 10.1016/j.chembiol.2014.06.009

Garcia-Santamarina, S., Uzarska, M. A., Festa, R. A., Lill, R., and Thiele, D. J. (2017). Cryptococcus neoformans iron-sulfur protein biogenesis machinery is a novel layer of protection against Cu Stress. MBio 8, 1-18. doi: 10.1128/mbio.01742-17

Gerwien, F., Skrahina, V., Kasper, L., Hube, B., and Brunke, S. (2018). Metals in fungal virulence. FEMS Microbiol. Rev. 42, 1-21. doi: 10.1093/femsre/fux050

Goswami, R. S., and Kistler, H. C. (2004). Heading for disaster: Fusarium graminearum on cereal crops. Mol. Plant Pathol. 5, 515-525. doi: 10.1111/J. 1364-3703.2004.00252.X

Grass, G., Rensing, C., and Solioz, M. (2011). Metallic copper as an antimicrobial surface. Appl. Environ. Microbiol. 77, 1541-1547. doi: 10.1128/AEM.02766-10

Iida, Y., Kurata, T., Harimoto, Y., and Tsuge, T. (2008). Nitrite reductase gene upregulated during conidiation is involved in macroconidium formation in Fusarium oxysporum. Phytopathology 98, 1099-1106. doi: 10.1094/PHYTO-9810-1099

Ilgen, P., Maier, F., and Schäfer, W. (2008). Trichothecenes and lipases are hostinduced and secreted virulence factors of Fusarium graminearum. Cereal Res. Commun. 36, 421-428. doi: 10.1556/CRC.36.2008.Suppl.B.35

Isebaert, S., Haesaert, G., Devreese, R., Maene, P., Fremaut, F., and Vlaemynck, G. (2005). Fusarium spp. and Fusarium mycotoxins in maize: a problem for Flanders? Commun. Agric. Appl. Biol. Sci. 70, 129-136.

Ji, F., Xu, J., Liu, X., Yin, X., and Shi, J. (2014). Natural occurrence of deoxynivalenol and zearalenone in wheat from Jiangsu province, China. Food Chem. 157, 393-397. doi: 10.1016/j.foodchem.2014.02.058

Keller, G., Bird, A., and Winge, D. R. (2005). Independent metalloregulation of Acel and Mac1 in Saccharomyces cerevisiae. Eukaryot. Cell. 4, 1863-1871. doi: 10.1128/EC.4.11.1863-1871.2005

Kim, B. E., Nevitt, T., and Thiele, D. J. (2008). Mechanisms for copper acquisition, distribution and regulation. Nat. Chem. Biol. 4, 176-185. doi: 10.1038/ nchembio.72

Kumar, S., Nei, M., Dudley, J., and Tamura, K. (2008). MEGA: a biologistcentric software for evolutionary analysis of DNA and protein sequences. Brief. Bioinform. 9, 299-306. doi: 10.1093/bib/bbn017

Ladomersky, E., and Petris, M. J. (2015). Copper tolerance and virulence in bacteria. Metallomics 7, 957-964. doi: 10.1039/c4mt00327f

Leslie, J. F., and Summerell, B. A. (2007). The Fusarium Laboratory Manual. Oxford: Blackwell Publishing. doi: 10.1002/9780470278376

Lombaert, G. A., Pellaers, P., Roscoe, V., Mankotia, M., Neil, R., and Scott, P. M. (2003). Mycotoxins in infant cereal foods from the Canadian retail market. Food Addit. Contam. 20, 494-504. doi: 10.1080/0265203031000094645
Lorenzo-Gutiérrez, D., Gómez-Gil, L., Guarro, J., Roncero, M. I. G., FernándezBravo, A., Capilla, J., et al. (2019). Role of the Fusarium oxysporum metallothionein $\mathrm{Mt1}$ in resistance to metal toxicity and virulence. Metallomics 11, 1230-1240. doi: 10.1039/c9mt00081j

Michels, H. T., Keevil, C. W., Salgado, C. D., and Schmidt, M. G. (2015). From laboratory research to a clinical trial: copper alloy surfaces kill bacteria and reduce hospital-acquired infections. Heal. Environ. Res. Des. J. 9, 64-79. doi: $10.1177 / 1937586715592650$

Odermatt, A., Suter, H., Krapf, R., and Solioz, M. (1993). Primary structure of two P-type ATPases involved in copper homeostasis in Enterococcus hirae. J. Biol. Chem. 268, 12775-12779. doi: 10.1016/j.brainres.2016.08.031

Palacios, Ò, Atrian, S., and Capdevila, M. (2011). Zn-and Cu-thioneins: a functional classification for metallothioneins? J. Biol. Inorg. Chem. 16, 9911009. doi: 10.1007/s00775-011-0827-2

Proctor, R. H., Hohn, T. M., and McCormick, S. P. (1995a). Reduced virulence of Gibberella zeae caused by disruption of a trichothecene toxin biosynthetic gene. Mol. Plant Microbe Interact. 8, 593-601. doi: 10.1094/MPMI-8- $\ 0593$

Proctor, R. H., Hohn, T. M., McCormick, S. P., and Desjardins, A. E. (1995b). Tri6 encodes an unusual zinc finger protein involved in regulation of trichothecene biosynthesis in Fusarium sporotrichioides. Appl. Environ. Microbiol. 61, 19231930. doi: 10.1128/aem.61.5.1923-1930.1995

Raffa, N., Osherov, N., and Keller, N. P. (2019). Copper utilization, regulation, and acquisition by Aspergillus fumigatus. Int. J. Mol. Sci. 20:1980. doi: 10.3390/ ijms20081980

Rasmussen, P. H., Ghorbani, F., and Berg, T. (2003). Deoxynivalenol and other Fusarium toxins in wheat and rye flours on the Danish market. Food Addit. Contam. 20, 396-404. doi: 10.1080/0265203031000082495

Solioz, M., and Odermatt, A. (1995). Copper and silver transport by CopB-ATPase in membrane vesicles of Enterococcus hirae. J. Biol. Chem. 270, 9217-9221. doi: $10.1074 /$ jbc. 270.16 .9217

Thiele, D. J. (2015). ACE1 regulates expression of the Saccharomyces cerevisiae metallothionein gene. Mol. Cell. Biol. 8, 2745-2752. doi: 10.1128/mcb.8.7. $\backslash 2745$

Thompson, J. D., Higgins, D. G., and Gibson, T. J. (1994). Clustal W: improving the sensitivity of progressive multiple sequence alignment through sequence weighting, position-specific gap penalties and weight matrix choice. Nucleic Acids Res. 22, 4673-4680. doi: 10.1093/nar/22.22.4673

Tutelyan, V. A. (2004). Deoxynivalenol in cereals in Russia. Toxicol. Lett. 153, 173-179. doi: 10.1016/j.toxlet.2004.04.042

Weissman, Z., Berdicevsky, I., Cavari, B.-Z., and Kornitzer, D. (2000). The high copper tolerance of Candida albicans is mediated by a P-type ATPase. Proc Natl Acad Sci U.S.A. 97, 3520-3525. doi: 10.1073/pnas.97.7.3520

Wiemann, P., Perevitsky, A., Huttenlocher, A., Osherov, N., and Keller, N. P. (2017). Aspergillus fumigatus copper export machinery and reactive oxygen intermediate defense counter host copper-mediated oxidative antimicrobial offense. Cell Rep. 19, 1008-1021. doi: 10.1016/j.celrep.2017.04.019

Wu, A. B., Li, H. P., Zhao, C. S., and Liao, Y. C. (2005). Comparative pathogenicity of Fusarium graminearum isolates from China revealed by wheat coleoptile and floret inoculations. Mycopathologia 160, 75-83. doi: 10.1007/s11046-0051153-4

Yang, K., Shadkchan, Y., Tannous, J., Landero Figueroa, J. A., Wiemann, P., Osherov, N., et al. (2018). Contribution of atpase copper transporters in animal but not plant virulence of the crossover pathogen Aspergillus flavus. Virulence 9 , 1273-1286. doi: 10.1080/21505594.2018.1496774

Yu, J. H., Hamari, Z., Han, K. H., Seo, J. A., Reyes-Domínguez, Y., and Scazzocchio, C. (2004). Double-joint PCR: a PCR-based molecular tool for gene manipulations in filamentous fungi. Fungal Genet. Biol. 41, 973-981. doi: 10.1016/j.fgb.2004.08.001

Conflict of Interest: The authors declare that the research was conducted in the absence of any commercial or financial relationships that could be construed as a potential conflict of interest.

Copyright (c) 2020 Liu, Jiang, He, Fang, Xu, Lee, Keller and Shi. This is an open-access article distributed under the terms of the Creative Commons Attribution License (CC BY). The use, distribution or reproduction in other forums is permitted, provided the original author(s) and the copyright owner(s) are credited and that the original publication in this journal is cited, in accordance with accepted academic practice. No use, distribution or reproduction is permitted which does not comply with these terms. 